\title{
Texture Recognition with combined GLCM, Wavelet and Rotated Wavelet Features
}

\author{
Dipankar Hazra
}

\begin{abstract}
Aim of this paper is to develop a texture recognition system for browsing and retrieval of image data. Many features have been proposed to precisely define the natural texture properties. Tamura proposed six features. Those features are coarseness, contrast, directionality, line-likeness, regularity and roughness. Haralick extract some features from gray level co-occurrence matrix (GLCM). Gabor features and wavelet features are widely used in image retrieval system and gives good result. In this paper combination of gray-level co-occurrence matrix, Daubechies filters and rotated wavelet filters are used to get a high quality feature set. A new algorithm is proposed to get rotated wavelet filter from Daubecheis wavelet coefficients. Experimental results demonstrate that the propose method is very efficient and superior to some other existing method.
\end{abstract}

Index Terms-Content based image retrieval, rotated wavelet filter, texture retrieval, wavelet.

\section{INTRODUCTION}

With the large growth of digital image libraries searching and browsing images with the conventional keyword based techniques are becoming obsolete. To attach a keyword with the image is very difficult task. Similarly using a keyword for searching is also difficult. Hence there is requirement for Content Based Image Retrieval (CBIR). It helps in visual queries. Features of images are stored as feature vector that is much smaller than original image. Image is searched by calculating minimum distance between feature vector of query image and same of the database image.

Most powerful features used in Content Based Image Retrieval are shape, texture and color of the images. Texture based objects are those objects for which unlike shape based objects, there is no visible inter-object part-wise correspondence. These objects are better described by their texture than the geometric structure of reliably detectable part. Buildings, roads, trees and skies are texture based objects. Sometimes when we are using color based segmentation shadow is detected as different object. But if we use texture based object detection technique shadow can be completely eliminated. For example, texture of vehicle is completely different from texture of road. Texture of road does not change when it is covered with shadow. Texture attribute of the road background is taken out. If texture attribute does not change or little changed then it is road, otherwise it is vehicle.

Many features have been proposed to precisely describe the natural texture properties. Tamura [1] proposed six

Dipankar Hazra is a Senior Lecturer in the department of Computer Science \& Engineering, Dr..B.C.Roy Engineering College, Fuljhore, Durgapur, West Bengal, India.(e-mail: dipankar1998@rediffmail.com). texture features namely coarseness, contrast, directionality, linelikeness, regularity and roughness. When two patterns differ only in scale the magnified one is coarser. For texture of different structure the bigger is the element size, less the elements are repeated is called coarser. Contrast can be seen as dynamic range of gray level or sharpness of edges etc. Directionality can be monodirectional or bidirectional. It is independent of orientation. Line-likeness is concerned only with shape of the texture element. Regularity is the variation of the property rule. Roughness is the total energy changes in the gray levels. It depends on coarseness and contrast.

Many texture based feature extraction methods are possible. Some of the most popular methods are gray level co-occurrence matrix [2], [3], gabor filters [4], haar filters [5], daubechies filters [6]-[9] etc. In this paper combination of gray level co-occurrence matrix, daubechies filters and rotated wavelet filters [7] have been used as feature extraction methods.

\section{RELATED WORKS}

\section{A. Gray Level Co-occurrence Matrix}

Distribution of pixel gray levels can be described by second-order statistics like the probability of two pixels having particular gray levels at particular spatial relationships. This information can be summarized in two dimensional gray level co-occurrence matrices, which can be computed for various distances and orientations. In order to use information contained in the GLCM, Haralick defined some statistical measures to extract textual characteristics. Some of these features are entropy, contrast, homogeneity, correlation etc. Contrast measures the local variation in the gray level of glcm. Correlation measures the joint probability of occurrence of pixel pairs of glcm. Energy gives the sum of squared pixel values of glcm. Homogeneity refers to the closeness of distribution of elements to the glcm diagonal. Homogeneous textures contain ideal repetitive structures. Weak homogeneity refers to variation in texture elements in their spatial arrangements.

The GLCM element C(i, j, d, $\Theta)$ represent probability of the pair of pixels, which are located with an inter sample distance $\mathrm{d}$ and a direction $\Theta$, have a gray level $\mathrm{i}$ and a gray level $\mathrm{j}$. The feature used in this work is Homogeneity. If the inter-pixel distance is set to 1 or $2 \mathrm{glcm}$ measure the local high frequency information.

\section{B. 2D Wavelet}

A wavelet is a mathematical function that is used to represent a continuous time signal into different scale components. A wavelet transform is representation of a 
function by wavelets. It has advantages over traditional Fourier transform for representing signal with discontinuities and sharp peaks and for accurately deconstructing and reconstructing signals.

The most known family of orthogonal wavelets is a family of Daubechies. Daubechies' wavelets are useful for texture classification and more popular due to their relations to multiresolution analysis (MRA). Daubechies' wavelets are usually denominated by the number of nonzero coefficients a_k, so usually talk about Daubechies 4, Daubechies 6 etc. wavelets. Roughly said, with the increasing number of wavelet coefficient the functions become more smooth.

Reference [6] shows that if $\phi(t)$ is the scaling function and $\psi(t)$ is the wavelet function then $\phi(t)$ satisfies the following:

It integrates to 1 .

$$
\int_{-\infty}^{\infty} \Phi(t) d t=1
$$

The scaling function has unit energy.

$$
\int_{-\infty}^{\infty}|\Phi(t)|^{2} d t=1
$$

The set consisting of $\phi(t)$ and its integer translates are orthogonal.

$$
<\Phi(t), \Phi(t-n)>=\delta(n)
$$

Wavelet function $\psi(t)$ satisfies the following:

It integrates to 0 .

$$
\int_{-\infty}^{\infty} \psi(t) d t=0
$$

The wavelet function has unit energy.

$$
\int_{-\infty}^{\infty}|\psi(t)|^{2} d t=1
$$

The set consisting of $\psi(t)$ and its integer translates are orthogonal.

$$
<\psi(t), \psi(t-n)>=\delta(n)
$$

The set consisting of $\psi(t)$ and $\phi(t)$ or its integer translates are orthogonal.

$$
<\psi(t), \Phi(t-n)>=0
$$

If Daubecheis 4 scaling function coefficients are $\mathrm{h}_{0}, \mathrm{~h}_{1}, \mathrm{~h}_{2}$, $h_{3}$ and wavelet function coefficients are $g_{0}, g_{1}, g_{2}, g_{3}$ then we obtain the following equations:

$$
\begin{gathered}
h_{0}^{2}+h_{1}^{2}+h_{3}^{2}+h_{4}^{2}=1 \\
h_{0} h_{2}+h_{1} h_{3}=0 \\
h_{0}+h_{1}+h_{2}+h_{3}=\sqrt{2} \\
g_{0}^{2}+g_{1}^{2}+g_{2}^{2}+g_{3}^{2}=1 \\
g_{0} g_{2}+g_{1} g_{3}=0 \\
g_{0}+g_{1}+g_{2}+g_{3}=0 \\
g_{1}+2 g_{2}+3 g_{3}=0
\end{gathered}
$$

Equation (8) and Equation (9) correspond to orthonormality of scaling functions. Equation (10) satisfies dilation equation. Similarly Equation (11) and Equation (12) correspond to orthonormality of wavelet function. Equation (14) gives moment of wavelet function. Solving the above equations we get following coefficient values:

$$
\begin{aligned}
& h_{0}=(1+\sqrt{3}) / 4 \sqrt{2} \\
& h_{1}=(3+\sqrt{3}) / 4 \sqrt{2} \\
& h_{2}=(3-\sqrt{3}) / 4 \sqrt{2} \\
& h_{3}=(1-\sqrt{3}) / 4 \sqrt{2}
\end{aligned}
$$

Since wavelet function is orthogonal to scaling function, scaling function and wavelet function coefficients are related as following:

$$
g_{0}=h_{3}, g_{1}=-h_{2}, g_{2}=h_{1}, g_{3}=-h_{0}
$$

The image is divided into 4 subbands by applying DWT as shown in Fig.1 (a). These subbands are labeled LH1, HL1, and $\mathrm{HH} 1$ represents finest scale wavelet coefficients while LL1 corresponds to coarse level coefficient. LL1 further decomposed to get next coarse level of wavelet coefficients. This results into two level wavelet decomposition as shown in Fig. 1 (b). This process is continued until some final scale is reached.

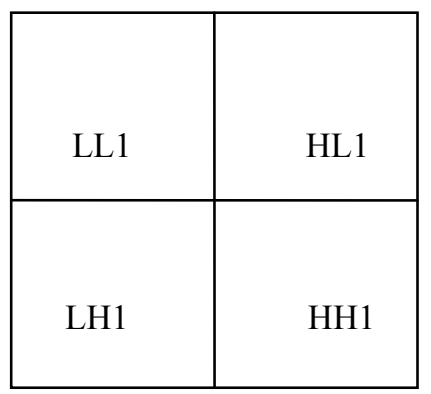

Fig.1 (a) 1-level Image Decomposition

\begin{tabular}{|c|c|c|}
\hline LL2 & HL2 & \multirow{2}{*}{ HL1 } \\
LH2 & HH2 & \\
\hline LH1 & HH1 \\
& \\
\hline
\end{tabular}

Fig.1 (b) 2-level Image Decomposition

\section{Rotated Wavelet Filter}

In texture retrieval application characterization of directional information improves texture retrieval performances. Rotated discrete wavelet filters can be used by rotating standard 2D discrete wavelet filters.

High-High subband in standard DWT decomposition contains the diagonal information of textured image. It is difficult to distinguish whether it is $45^{\circ}$ or $135^{\circ}$. Hence rotated wavelet filter has been used. Here Daubecheis 4 filter coefficients are oriented at angle $\Theta$ and convolved with the textured image at multiple resolutions. The value of $\Theta$ can be $0^{\circ}$ to $180^{\circ}$ to retrieve textured information on that specific 
direction. In this experiment $\Theta$ has been taken as $45^{\circ}$. Low-High and High-Low subbands are mainly containing diagonal information. Hence only energy of these two subbands at multiple resolutions has been considered.

\section{PROPOSED APPROACH}

\section{A. Texture Recognition System}

Fig.2 illustrates the proposed Texture Recognition System. This system is composed of two sub-systems or components:

(i) texture database

(ii) texture recognition function

\section{Texture Database:}

Each texture name, texture type and glcm feature vector is stored in a table for glcm texture database.

Similarly, each texture name, texture type and 2D wavelet feature vector and rotated wavelet filter feature vector is stored in a table for wavelet texture database.

\section{Texture Recognition Function:}

This function calculates and stores the feature vectors of training images. It also calculates feature vectors of the query image and compares with the feature vectors of training images. It classifies the query image. This function consists of two sub function. One is for gray level co-occurrence matrix and another is for standard wavelet and rotated wavelet filter.

\section{B. Texture Features}

For GLCM Homogeneity are calculated for four direction (i.e. $\Theta=0^{\circ}, 45^{\circ}, 90^{\circ}$ or $135^{\circ}$ ). A feature vector of size 4 is created for the image. Homogeneity is computed as follows,

$$
\text { Homogeneity }=\sum_{i, j} \frac{p(i, j)}{1+|i-j|}
$$

The DWT decomposition of the image is performed up to 5 th level. Energy for each level, for each sub band (High-High, High-Low, Low-High, Low-Low) are calculated and taken as feature vector of size $5 \times 4=20$. Energy is computed as follows,

$$
\text { Energy }=\frac{1}{M \times N} \sum_{i=1}^{M} \sum_{j=1}^{N}\left|X_{i j}\right|
$$

Similarly RWF decomposition of the image is performed up to 5 th level. Energy for each level, for Low-High and High-Low subband are calculated to extract the diagonal texture features using the same formula above. This gives feature vector of size $5 \times 2=10$.

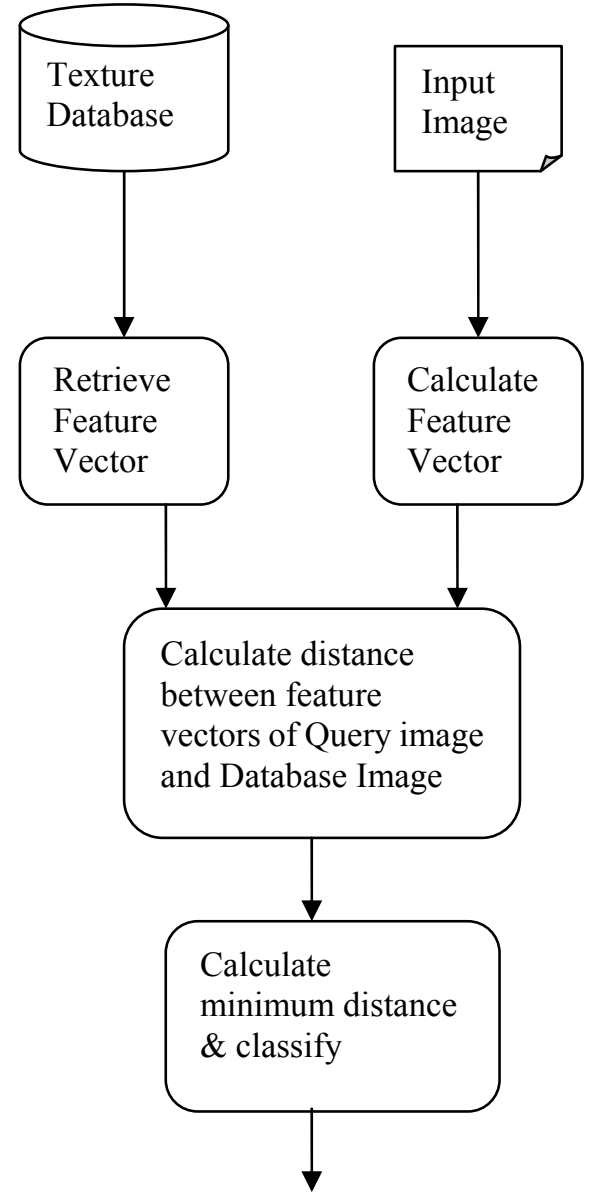

Fig.2 Proposed Texture Recognition System

\section{RWF Algorithm}

Following algorithm has been used to orient Daubechies 4 coefficients at angle $\Theta$ for creating rotated wavelet filter. Let lpf and hpf represent the one dimensional Daubechies 4 low pass and high pass filter coefficients respectively.

$$
\operatorname{lpf}=\left[\begin{array}{llll}
h_{0} & h_{1} & h_{2} & h_{3}
\end{array}\right], h p f=\left[\begin{array}{llll}
g_{0} & g_{1} & g_{2} & g_{3}
\end{array}\right] .
$$

Let rot lpf and rot hpf represent the rotated wavelet lowpass and high pass filters coefficients respectively. In the proposed algorithm the rot_lpf and rot_hpf using $\Theta=45^{\circ}$ will be as following:

$$
\begin{aligned}
& \operatorname{rot} \operatorname{lpf}=\left(\begin{array}{llll}
\mathrm{h}_{0} & 0 & 0 & 0 \\
0 & \mathrm{~h}_{1} & 0 & 0 \\
0 & 0 & \mathrm{~h}_{2} & 0 \\
0 & 0 & 0 & \mathrm{~h}_{3}
\end{array}\right) \\
& \operatorname{rot} \mathrm{hpf}=\left(\begin{array}{llll}
\mathrm{g}_{0} & 0 & 0 & 0 \\
0 & \mathrm{~g}_{1} & 0 & 0 \\
0 & 0 & \mathrm{~g}_{2} & 0 \\
0 & 0 & 0 & \mathrm{~g}_{3}
\end{array}\right)
\end{aligned}
$$

Step I: $\quad$ Set lpf to Daubechies 4 scaling coefficients; 
Step II: $\quad$ lf= length(lpf);

Step III: $\quad$ FOR $\mathrm{i}=1$ to $1 \mathrm{f}-1$

COMPUTE

$\operatorname{hpf}(i+1)=(-1)^{\wedge} i * \operatorname{lpf}(1 \mathrm{f}-\mathrm{i})$

END FOR;

Step IV: $\quad$ SET $\Theta$ to rotation of wavelet filter;

Step $V: \quad$ COMPUTE

$\mathrm{m}=\operatorname{tangent}(\Pi / 2-\theta)$;

Step VI: $\quad$ IF ( $\mathrm{m}$ is less than 1$)$

SET dx to 1;

SET dy to m;

ELSE

SET dy to 1;

SET $d x$ to $1 / m$;

END IF;

Step VII: SET xa to 1;

SET ya to 1 ;

SET rot $\operatorname{lpf}($ xa,ya) to $\operatorname{lpf}(1)$;

SET rot_hpf(xa,ya) to $h p f(1)$;

Step VIII: FOR i 1 to (lf-1)

COMPUTE $\mathrm{xa}=\mathrm{xa}+\mathrm{dx}$;

COMPUTE $y a=y a+d y$;

COMPUTE rxa=round(xa);

COMPUTE rya=round(ya);

SET rot_lpf(rxa,rya) to $\operatorname{lpf}(\mathrm{i}+1)$;

SET rot hpf(rxa,rya) to hpf(i+1);

END FOR;

\section{Texture Recognition}

The feature vector of the query image is compared with the feature vector of the database image. It then classifies the image according to minimum distance. Let $\mathrm{x}$ and $\mathrm{y}$ are feature vectors of two images.

$$
\begin{aligned}
& x=\left[\begin{array}{lllll}
x_{1} & x_{2} & x_{3} & \ldots \ldots . . & x_{d}
\end{array}\right] \\
& y=\left[\begin{array}{llllll}
y_{1} & y_{2} & y_{3} & \ldots & \ldots . . . y_{d}
\end{array}\right]
\end{aligned}
$$

$\mathrm{x}_{1}, \mathrm{x}_{2}, \mathrm{x}_{3}, \ldots \ldots, \mathrm{x}_{\mathrm{d}}$ are energy of different subbands ( LL, $\mathrm{LH}, \mathrm{HL}, \mathrm{HH}$ ) of different levels of wavelet decomposition. Similarly, $\mathrm{y}_{1}, \mathrm{y}_{2}, \mathrm{y}_{3}, \ldots . . ., \mathrm{y}_{\mathrm{d}}$ are energy of different subbands ( LL, LH, HL,HH) of different levels of wavelet decomposition.

Distance between $\mathrm{x}, \mathrm{y}$ is as following:

Manhattan Distance

$$
\operatorname{disman}(x, y)=\sum_{i=1}^{d}\left|x_{i}-y_{i}\right|
$$

\section{Euclidean Distance}

$$
\operatorname{diSeucl}(x, y)=\sqrt{\sum_{i=1}^{d}\left(x_{i}-y_{i}\right)^{2}}
$$

$$
\begin{aligned}
& \text { Canberra Distance } \\
& \qquad \operatorname{discanb}(x, y)=\sum_{i=1}^{d} \frac{\left|x_{i}-y_{i}\right|}{\left|x_{i}+y_{i}\right|}
\end{aligned}
$$

Euclidean Distance is mostly used. Each dimension is squared before summation in Euclidean distance method. It is more useful when dissimilarity is large [7], whereas Canberra Distance appears to be good similarity measure to be used which normalizes the difference to avoid scaling effect.

\section{EXPERIMENTAL RESULT}

The images are obtained from the Brodatz image database [10]. Experiments are conducted with 5 types of images with 7 orientations $\left(0^{\circ}, 30^{\circ}, 60^{\circ}, 90^{\circ}, 120^{\circ}, 150^{\circ} \& 200^{\circ}\right) .21 \mathrm{sub}$ image of size $64 \times 64$ are obtained from each image to form the textured image database. Texture Database consists of the following datasets.

Water Dataset: $7 \times 21$ textures have been taken as training dataset.

Sand Dataset: $7 \times 21$ textures have been taken as training dataset.

Grass Dataset: $7 \times 21$ textures have been taken as training dataset.

Wood Dataset: $7 \times 21$ textures have been taken as training dataset.

Pigskin Dataset: $7 \times 21$ textures have been taken as training dataset.

Fig. 3 shows sample texture images of each type. 5 types of images with 7 orientations with size $64 \times 64$ have been considered as query image. Hence total numbers of query images are $5 \times 7=35$. Fig. 4 shows 10 similar most images for a query image. Water.000.tiff is an image of water with $0^{\circ}$ orientation. A subimage of size $64 \times 64$ of water.000.tiff has been used as the query image with top left coordinate at $(257,257)$. Following the query image, there are10 similar images of size $64 \times 64$. Image name and the co-ordinate of the top left corner of that image are specified below each image.

From Table I it is observed that combination of GLCM, Daubechies 4 Wavelet, and Rotated Wavelet outperforms other two methods. But retrieval accuracy of different textures is different. This is shown in Table II.

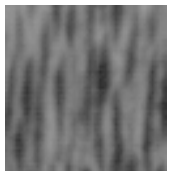

Water.000.tiff Sand.030.tiff
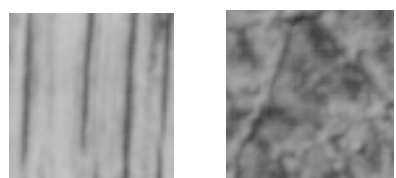

Wood.000.tiff Pigskin.200.tiff
Query Image 


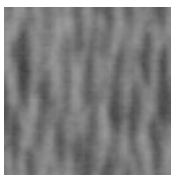

(water.000.tiff, 257,257)

Top 10 similar images

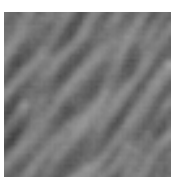

(water.150. tiff, 1,257)

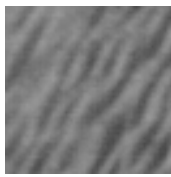

(water.150. tiff, 129,129)

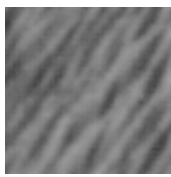

(water.150. tiff, 129,385)

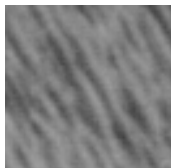

(water.030.tiff, 129,257)

Fig.4 Query image and 10 similar most images of query image of size $64 \times 64$.

TABLE I ACCURACY TABLE FOR DIFFERENT METHOD.

\begin{tabular}{|c|c|c|}
\hline $\begin{array}{c}\text { Daubechie } \\
\text { s 4 Wavelet }\end{array}$ & $\begin{array}{c}\text { Daubechies 4 } \\
\text { Wavelet \& } \\
\text { Rotated Wavelet }\end{array}$ & $\begin{array}{c}\text { GLCM \& Daubechies } \\
\text { 4 Wavelet \& Rotated } \\
\text { Wavelet }\end{array}$ \\
\hline $65.71 \%$ & $77.14 \%$ & $85.71 \%$ \\
\hline
\end{tabular}

Table II Accuracy Table for Different Textures using GLCM, Daubechies 4 Wavelet and Rotated Wavelet.

\begin{tabular}{|l|l|l|l|l|}
\hline Wood & Grass & Water & Sand & Pigskin \\
\hline $100 \%$ & $100 \%$ & $85.71 \%$ & $85.71 \%$ & $57.14 \%$ \\
\hline
\end{tabular}

\section{Conclusion}

In this research an efficient texture based object recognition algorithm is proposed. The result shows that the approach has given good result comparing to other existing texture recognition algorithm. We have used energy feature of wavelet subbands to classify image. Further research can be done to improve retrieval accuracy by using other features of wavelet subbands. Length of the wavelet filters can also be increased to get better result. Also the proposed Rotated Wavelet Filter algorithm can be applied for other problems like noise removal of an image.

\section{REFERENCES}

[1] H Tamura, S Mori, T Yamawaki, "Textural Features Corresponding to Visual Perception", IEEE Transaction on Systems, man and Cybernetics, Vol. 8, No. 6, 1978W.-K. Chen, Linear Networks and Systems (Book style). Belmont, CA: Wadsworth, 1993, pp. 123-135.

[2] R.M. Haralick, K. Shanmugam, I. Dinstein, "Textural Features for Image Classification", IEEE Transaction on Systems, man and Cybernetics, Vol. SMC-3, pp.610-621, 1973.B. Smith, “An approach to graphs of linear forms (Unpublished work style)," unpublished.

[3] Shankar Bhausaheb Nikam, Suneeta Agarwal, "Wavelet Energy Signature and GLCM Features-based fingerprint anti-spoofing", Proceedings of the 2008 International Conference on Wavelet Analysis and Pattern Recognition, Hong Kong, 30-31 Aug,2008.

[4] Manjunath B.S, Ma W.Y, "Texture features for browsing and retrieval of image data", IEEE Transactions on Pattern Analysis and Machine Intelligence, Vol. 18, No. 8, Aug, 1996.

[5] Q Tian, N Sebe, M S Lew, E Loupias, T S Huang, “ Image Retrieval using wavelet-based salient points", Journal of Electronic Imaging, Special Issue on Storage and Retrieval of Digital Media, pp.835-849, vol.10(4),Oct, 2001.

[6] Raghuveer M. Rao and Ajit S. Bopardikar, Wavelet Transforms: Introduction to Theory \& Application, Pearson Education, India, 2009, pp. 66-73.

[7] M. Kakore, P.K. Biswas, B.N. Chatterjee, "Texture image retrieval using rotated wavelet filters", Pattern Recognition Letters 28, 1240-1249, 2007.

[8] K. Muneeswaran, L. Ganeshan, S.Arumugam and K. Ruba Soundar, "Texture classification with combined rotation and scale invariant wavelet features", Pattern Recognition 38,1495-1506, 2005.

[9] Tianhorng Chang and C C Jay Kuo, "Texture Analysis \& Classification with Tree-Structured Wavelet Transform", IEEE Transaction on Image Processing, vol-2, no 4, 1993.

[10] P. Brodatz, Textures: A Photographic Album for Artists and Designers, Dover, New York, 1966.

Dipankar Hazra received the B.C.S.E. degree and M.TECH-IT (Courseware Engineering) degree both from Jadavpur University in 1998 and 2009 respectively. He is a Senior Lecturer at the Department of Computer Science and Engineering at Dr.B.C.Roy Engineering College, Fuljhore, Durgapur,West Bengal, India. His research interests include DBMS, Data Mining and Image Processing. 